\title{
Morbidade referida, situação vacinal e acesso a serviços de saúde por pré-escolares*
}

\author{
Reported morbidity, vaccination status and access to health services \\ in early childhood
}

Patricia L. dos Santos ${ }^{1}$

\begin{abstract}
RESUMO
Modelo de estudo: estudo de prevalência. Objetivos do estudo: Identificar os principais motivos de busca de atendimento nos serviços de saúde básica de crianças de três a seis anos de idade, bem como verificar a situação vacinal dessas crianças e a frequência com que buscam a unidade de saúde. Metodologia: Foram analisados 370 prontuários de crianças com idades entre três e seis anos, atendidas em quatro Núcleos de Saúde da Família e uma Unidade Básica de Saúde, colhendo-se informações sobre sexo, idade, número de consultas nos últimos 12 meses, situação vacinal e queixas apresentadas. As queixas foram organizadas em 28 categorias de acordo com o problema ou o órgão atingido. Foram calculadas frequência e porcentagem de todas as queixas e calculados os índices de consultas ao ano e cobertura vacinal. Resultados: As queixas que ocorreram com maior frequência foram: tosse, febre, problemas no nariz, problemas na pele ou couro cabeludo, problemas digestivos, queda de apetite ou perda de peso, problemas emocionais ou comportamentais, dores abdominais, problemas respiratórios ou pulmonares, problemas no ouvido. Apenas $3,2 \%$ das crianças estavam com suas vacinas atrasadas e o número médio de consultas foi de 3,56. Conclusões: Embora a tendência no atendimento básico em saúde seja enfatizar os aspectos de prevenção e promoção de saúde, ainda há um privilégio da dimensão física no olhar dos profissionais e na busca pelos serviços. Faz-se necessária maior atenção aos aspectos psicossociais da saúde.
\end{abstract}

Palavras-chave: Saúde Infantil. Saúde Pública. Morbidade. Perfil de saúde.

\section{Introdução}

No Brasil, nos últimos 20 anos, tem havido um grande investimento no setor da saúde, priorizando ações básicas, direcionando cada vez mais o setor para atividades de prevenção e promoção de saúde.
Mais especificamente com relação à saúde da criança, o Ministério da Saúde e as secretarias Estaduais e Municipais de Saúde, com o apoio de entidades internacionais e filantrópicas, têm implementado programas voltados aos problemas de alta morbidade e mortalidade.
* Saúde de crianças de 3 a 6 anos / Early Childhood Health

1. Docente; Departamento de Neurologia, Psiquiatria e Psicologia Médica; Faculdade de Medicina de Ribeirão Preto da Universidade de São Paulo.

Auxílio pesquisa - FAEPA (Fundação de Amparo ao Ensino, Pesquisa e Assistência do Hospital das Clínicas da FMRPUSP).
Correspondência: Rua Ten. Catão Roxo, 2650, sala 32

Vila Monte Alegre, 14051-140 - Ribeirão Preto/SP Tel.: (16) 36024608 Fax: (16) 36024504 E-mail: plsantos@fmrp.usp.br

Artigo recebido em 16/09/2008 Aprovado em 05/03/2009 
Assim, os programas de atenção à saúde da criança têm desenvolvido atividades voltadas, principalmente, para o incentivo ao aleitamento materno, cobertura vacinal, controle de diarréia e infecções respiratórias agudas, com foco especialmente sobre as crianças menores de cinco anos. ${ }^{1}$

Embora os estudos e as ações estejam mais centralizados na dimensão física/orgânica e focalizem principalmente crianças de zero a cinco anos de idade, mais suscetíveis à morte por diarréia, pneumonia e malnutrição, ${ }^{2-5}$ é cada vez mais presente a noção de que a saúde é o resultado de múltiplas combinações entre diferentes variáveis que englobam desde o aspecto biológico, humano e social até as condições políticas, econômicas e educacionais.

Os estudos também têm procurado avaliar o alcance e cobertura dos diferentes programas de vigilância e promoção da saúde infantil.

Nesta direção, Santos et $\mathrm{al}^{1}$, encontraram um bom índice de cobertura dos programas de vacinação, puericultura, assistência pré-natal e saúde da mulher, mas observaram problemas quanto à monitorização do crescimento infantil, consultas de revisão de parto, realização de exames preventivos de câncer de útero e mama. Os principais motivos para a consulta médica de crianças menores de um ano, identificados pelos autores foram: puericultura/consulta de rotina (66\%) e infecções respiratórias agudas (IRA - 26,8\%).

Outros autores têm destacado a importância do estado nutricional para a saúde da criança, pois, embora tenha havido um decréscimo importante nas taxas de desnutrição infantil nas últimas três décadas, ainda é alto o índice de crianças anêmicas em alguns estados brasileiros. A ocorrência de anemia tem sido associada com condições sócio-econômicas, condições de assistência à saúde da criança, o estado nutricional geral, presença de doenças, hábitos de consumo alimentar e fatores biológicos. ${ }^{2,6,7}$

Estudos têm mostrado, também, que famílias de menor poder aquisitivo retardam a procura de atendimento adequado para seus filhos, prejudicando o estado de saúde dos mesmos, favorecendo o agravamento da doença e a hospitalização. ${ }^{5}$

$\mathrm{Na}$ última década, novas políticas públicas foram construídas e os investimentos foram ampliados para promover a organização da atenção básica nos municípios, desenvolvendo-se os Programas de Agentes Comunitários de Saúde e Saúde da Família, com o objetivo principal de levar a saúde para mais perto das famílias, melhorando a qualidade de vida da população. Busca-se assim, que os serviços e a população partilhem a responsabilidade sobre a cura, a prevenção e a promoção de saúde. ${ }^{8,9}$

Um dos aspectos a serem avaliados para analisar o alcance dos objetivos propostos pelo Programa de Saúde da Família é a análise dos motivos e frequência de utilização do serviço, tanto quanto a facilidade de acesso, a visão que o usuário tem do serviço, o momento em que o atendimento é procurado, a participação nas atividades preventivas. ${ }^{10}$

É possível que a reorganização dos serviços de atendimento básico à saúde, especialmente com a implantação do Programa de Saúde da Família, facilitando o acesso aos serviços, tenha alterado de alguma maneira o perfil de morbidade desta população, destacando problemas para os quais as famílias não estavam atentas anteriormente.

A estratégia de Saúde da Família, tendo como foco a família, busca a integração dos serviços e equipes de saúde com a comunidade, bem como realizar uma busca ativa de casos para intervenção, enfatizando a prevenção e promoção de saúde. Dessa forma ela amplia o olhar sobre a saúde, além de facilitar o acesso aos serviços e orientações. ${ }^{11}$

A partir da análise dos registros dos serviços de saúde, este trabalho teve como objetivo identificar os principais motivos de busca de atendimento nos serviços básicos de saúde de crianças de três a seis anos de idade, bem como verificar a situação vacinal dessas crianças e a frequência com que buscam a unidade de saúde.

\section{Metodologia}

Este projeto foi aprovado pelo Comitê de Ética do Centro de Saúde Escola da Faculdade de Medicina de Ribeirão Preto da Universidade de São Paulo e, faz parte de um estudo maior que visa conhecer as condições de saúde e desenvolvimento das crianças de três a sete anos de idade, observando quais os problemas que não geram a busca do serviço ou que não são identificados pelas equipes de saúde, com especial atenção à saúde mental na infância.

Trata-se de um estudo transversal e descritivo para o qual foram analisados 370 prontuários de crianças com idades entre três e seis anos, atendidas em quatro Núcleos de Saúde da Família e uma Unidade Básica e Distrital de Saúde (que atende a uma micro área que ainda não tem um Núcleo ou Equipe de Saú- 
de da Família instalado) de um distrito de saúde do município de Ribeirão Preto, interior de São Paulo.

Estas crianças estão há pelo menos um ano fora da rotina de puericultura (de zero a dois anos), entretanto, encontram-se ainda num período bastante sensível de seu desenvolvimento físico, cognitivo, emocional e social.

Para facilitar a coleta de dados, elaborou-se um protocolo que continha itens sobre sexo, idade, número de consultas nos últimos 12 meses, situação vacinal, queixas apresentadas, diagnóstico, orientações, exames solicitados e medicações prescritas. Para este trabalho foram analisados apenas os dados referentes aos cinco primeiros itens.

Para identificação dos prontuários que comporiam a amostra, foi feito um levantamento nos arquivos das unidades de saúde participantes, identificando-se todos os prontuários de crianças na faixa etária estudada. Foram excluídas da amostra as crianças que não haviam passado por nenhuma consulta nos 12 meses precedentes à coleta de dados (16\% dos prontuários).

Procurou-se anotar as informações exatamente como elas estavam registradas nos prontuários. Após a coleta foram listadas todas as queixas, que foram então agrupadas em categorias, de acordo com a queixa apresentada ou o órgão atingido pelo problema.

Após a elaboração das categorias as queixas foram classificadas e os dados foram armazenados para análise. A análise estatística foi feita com o auxílio do programa SPSS 10.0 para Windows.

Foram calculadas frequência e porcentagem de todas as queixas e verificado se haviam diferenças significativas entre os sexos e idades.

\section{Resultados}

Pela análise dos prontuários observou-se que a maioria das crianças atendidas nas unidades de saúde onde se realizou o estudo são meninos $(53,8 \%)$, predominando crianças com três e quatro anos de idade (52,9\% - Tabela 1$)$.

Quanto à situação vacinal, embora apenas 3,2\% das crianças estivessem com suas vacinas atrasadas, um alto índice de prontuários não continha esta informação (28,9\% - Tabela 2). Além disso, pode-se observar que à medida que as crianças ficam mais velhas aumenta o índice de atraso de vacinas (de 1,8\% aos três anos para 6,2\% aos seis anos).
Tabela 1

Distribuição da amostra por sexo, idade

\begin{tabular}{clcc}
\hline Variável & Frequência & Porcentagem \\
\hline Sexo & Feminino & 171 & 46,2 \\
& Masculino & 199 & 53,8 \\
Idade & 3 anos & 110 & 29,7 \\
& 4 anos & 86 & 23,2 \\
5 anos & 93 & 25,1 \\
6 anos & 81 & 21,9 \\
Total & 370 & 100,0 \\
\hline
\end{tabular}

\section{Tabela 2}

Distribuição da situação vacinal por idades (em porcentagem)

\begin{tabular}{|c|c|c|c|c|c|}
\hline & 3 anos & 4 anos & 5 anos & 6 anos & Total \\
\hline Vacinas em dia & 71,8 & 66,3 & 72,0 & 59,3 & 67,8 \\
\hline Vacinas atrasadas & 1,8 & 2,3 & 3,2 & 6,2 & 3,2 \\
\hline Sem informação & 26,4 & 31,4 & 24,7 & 34,6 & 28,9 \\
\hline
\end{tabular}

O número de consultas pelas quais as crianças tinham passado no último ano variou de uma a 17 consultas, sendo que $50 \%$ das crianças tiveram entre uma e três consultas e $25 \%$ tiveram mais de cinco consultas no ano. Nota-se na Tabela 3 que conforme as crianças ficam mais velhas diminui a média de consultas realizadas (de 4,48 consultas aos três anos para 2,9 aos seis anos).

\section{Tabela 3}

Distribuição das médias, desvio-padrão e medianas de consultas por idade

\begin{tabular}{lccccc}
\hline & 3 anos & $\mathbf{4}$ anos & $\mathbf{5}$ anos & $\mathbf{6}$ anos & Total \\
\hline Média & 4,48 & 3,51 & 3,10 & 2,90 & 3,56 \\
Desvio-padrão & 3,41 & 2,77 & 2,58 & 2,79 & 2,99 \\
Mediana & 4,00 & 3,00 & 2,00 & 2,00 & 3,00 \\
\hline
\end{tabular}

Quanto às queixas, foram encontradas 28 categorias, a saber: tosse; febre; problemas no nariz (coriza, rinorréia, coceira, obstrução, sangramento); problemas na pele ou couro cabeludo (coceira, escamação, lesões na pele, queda de pelos/cabelos); proble- 
mas digestivos (vômito, náuseas, mal-estar pós-alimentação); queda de apetite ou perda de peso; problemas emocionais ou comportamentais (irritação, nervoso, agitação, choro fácil, medos, entre outros); dores abdominais; problemas respiratórios ou pulmonares (falta de ar, respiração bucal, crise de bronquite, esforço respiratório); problemas no ouvido (dor de ouvido, secreção, coceira); problemas na garganta (dor, infecção, rouquidão); aumento de evacuações ou diarréia; problemas nos olhos (hiperemia, secreção, coceira, dor); queda do estado geral, abatimento, canseira; dor de cabeça; problemas na boca ou nos dentes (aftas, cáries, dor, feridas); problemas nos genitais (coceira, secreção, lesões, irritações); prurido anal; problemas de fala e/ou linguagem (fala errado, atraso no desenvolvimento da fala, gagueira); diminuição das evacuações ou constipação intestinal; gripe ou resfriado; ferimentos, cortes ou queimaduras; quedas ou traumas; déficit auditivo (não escuta direito, perda auditiva); dificuldade para andar, dor nas pernas ou joelho e caroços ou gânglios aumentados.

As queixas que ocorreram com maior frequência para o total da amostra foram: tosse $(46,5 \%)$, febre $(42,7 \%)$, problemas no nariz $(37,8 \%)$, problemas na pele ou couro cabeludo $(25,1 \%)$, problemas digestivos $(24,6 \%)$, queda de apetite ou perda de peso $(24,3 \%)$, problemas emocionais ou comportamentais $(23,5 \%)$, dores abdominais $(20,3 \%)$, problemas respiratórios ou pulmonares $(16,2 \%)$, problemas no ouvido $(15,4 \%)$ e problemas na garganta $(15,4 \%)$, aumento de evacuações ou diarréia (14,3\%), problemas nos olhos $(12,4 \%)$ e queda do estado geral, abatimento, canseira $(10,8 \%)$ (Tabela 4).

\section{Tabela 4}

Febre

Dor de cabeça

Prurido anal

Déficit auditivo
Verifica-se que apenas 3\% das crianças foram à unidade de saúde para um retorno regular ou de seguimento (após 6 meses ou 1 ano), sem apresentar qualquer queixa.

Não foi possível classificar 2,2\% dos registros encontrados nos prontuários, pois não especificavam
Distribuição das queixas apresentadas pelas crianças

\begin{tabular}{lcc}
\hline Queixa & Frequência & \% \\
\hline Tosse & 172 & 46,5
\end{tabular}
158 42,7

Problemas no nariz 37,8

Problemas na pele/couro cabeludo 25,1

Problemas digestivos 24,6

Queda de apetite/perda de peso 24,3

Problemas emocionais ou comportamentais

Dores abdominais 23,5

Problemas respiratórios/pulmonares 20,3

Problemas no ouvido 16,2

Problemas na garganta

Aumento de evacuações/diarréia

Problemas nos olhos

Queda do estado geral ou abatimento, canseira

Problemas na boca/nos dentes

Problemas nos genitais

Problemas de fala/linguagem

Diminuição das evacuações ou constipação

Gripe ou resfriado 15,4 57 15,4

Ferimentos, cortes, queimaduras

Muco, sangue, verme nas fezes

Problemas com a urina 14,3 53 12,4 46 10,8

40

Quedas ou traumas 7,5

Dificuldade para andar, dor nas pernas ou joelho

Caroços ou gânglios aumentados 
o problema de saúde nem o órgão atingido, sendo muito vagos ou incompletos.

Quanto às diferenças significativas observadas, tosse, problemas no nariz e problemas na boca ou dente foram mais frequentes entre os meninos, enquanto prurido anal e problemas com a urina ocorreram mais entre as meninas (Tabela 5).

Na Tabela 5 podem-se observar também as diferenças significativas entre as idades, notando-se que tosse; febre; problemas no nariz; queda de apetite ou perda de peso; dores abdominais; aumento de evacuações ou diarréia; queda do estado geral, abatimento, canseira; diminuição das evacuações ou constipação; gripe ou resfriado e quedas ou traumas ocorreram mais frequentemente entre as crianças mais novas (de três ou quatro anos).

\section{Discussão}

Embora a metodologia utilizada tenha limitações por se basear nos registros do serviço, ela permitiu identificar algumas questões quanto à saúde da criança que merecem atenção.

Diferentemente de outros estudos, neste trabalho optou-se por analisar as queixas referidas pelos responsáveis pelas crianças. Esta opção foi feita com o objetivo de identificar quais os problemas que geram a consulta, que muitas vezes são diferentes do que o médico detecta ao longo da sua avaliação.

Os dados sobre situação vacinal apontam uma falha importante nos registros, dado o alto índice de prontuários que não continha informações sobre vacinação.

\section{Tabela 5}

Diferenças significativas encontradas por sexo e por idade

\section{Diferenças por sexo (Teste Qui-quadrado de Pearson)}

\begin{tabular}{lccc} 
& Feminino & Masculino & P \\
\hline Tosse & 65 & 107 & 0,002 \\
Problemas no nariz & 53 & 87 & 0,012 \\
Problemas na boca ou dentes & 9 & 26 & 0,011 \\
Prurido anal & 16 & 7 & 0,020 \\
Problemas com a urina & 10 & 3 & 0,024
\end{tabular}

Diferenças significativas por idade (Teste Mann-Whitney)

\begin{tabular}{lcccc} 
& Média & Desvio-padrão & Mediana & P \\
\hline Tosse & 4,27 & 1,12 & 4,00 & 0,049 \\
Febre & 4,13 & 1,13 & 4,00 & 0,000 \\
Problemas no nariz & 4,23 & 1,14 & 4,00 & 0,028 \\
Queda de apetite/perda de peso & 4,11 & 1,11 & 4,00 & 0,006 \\
Dores abdominais & 4,11 & 1,16 & 4,00 & 0,013 \\
Aumento de evacuações/diarréia & 4,04 & 1,19 & 4,00 & 0,011 \\
Queda do estado geral, abatimento, canseira & 3,95 & 1,15 & 3,00 & 0,007 \\
Diminuição das evacuações ou constipação & 3,39 & 0,70 & 3,00 & 0,000 \\
Gripe ou refriado & 3,65 & 1,06 & 3,00 & 0.005 \\
Quedas ou traumas & 3,73 & 1,01 & 3,00 & 0,046 \\
\hline
\end{tabular}


Por outro lado, ao considerar o índice de crianças com vacinas atrasadas, podemos interpretar que haja uma boa cobertura, pelo menos entre as crianças que têm acesso aos serviços de saúde.

Dados nacionais mostram uma variação entre $65 \%$ e $100 \%$ de cobertura vacinal para crianças menores de um ano. ${ }^{12}$

Concordando com a literatura, ${ }^{13}$ os resultados apontaram que o atraso nas vacinas tende a ocorrer com maior frequência para as crianças mais velhas.

É provável que a maior frequência das crianças mais novas na unidade de saúde para a puericultura favoreça a vacinação, seja porque a criança já está no serviço, seja porque a mãe é lembrada sobre a importância da vacinação.

Há que se considerar que a maior parte da população atendida nos serviços em que este estudo foi realizado apresenta características descritas na literatura como risco para não vacinação, tais como baixa renda e baixa escolaridade. ${ }^{13}$

Além disso, no caso dos Núcleos de Saúde da Família, o paciente tem que se dirigir a outra unidade para tomar a vacina, aumentando o risco da não vacinação pela necessidade de deslocamento.

O Programa de Saúde da Família pela proximidade com a comunidade tem um papel importante na busca ativa e orientação sobre a importância da vacinação. Entretanto, depende da família levar a criança para tomar a vacina. $\mathrm{O}$ índice de atraso das vacinas para as crianças de seis anos se destaca, sendo importante insistir na notificação e agendamento de vacinas para as mesmas.

Outra questão a ser considerada é que o controle quanto à vacinação está em parte sob a responsabilidade das escolas, uma vez que o cartão de vacina é exigido no município para a efetivação da matrícula e, profissionais da saúde visitam as escolas fazendo o levantamento da situação vacinal dos alunos, sendo eles os responsáveis pela notificação das famílias e convocação para a vacinação nos casos de atraso.

Quanto ao uso dos serviços, como seria de se esperar, o número de consultas realizadas no período de um ano tende a diminuir conforme as crianças ficam mais velhas. Entretanto, merece atenção o fato de que um quarto da amostra tenha tido cinco ou mais consultas, como também a ocorrência de crianças que tiveram mais de 10 consultas no ano.

É importante avaliar esses casos e ampliar os esforços para a prevenção dos problemas de saúde na infância. Estas crianças mereceriam estar sendo acompanhadas mais de perto pelas equipes de saúde, entretanto, esta rotina não está prevista nos programas.

A recorrência frequente aos serviços alerta para uma questão pouco olhada pela maioria dos profissionais de saúde: as condições de risco ao desenvolvimento infantil. Episódios recorrentes de doença interferem na rotina da criança, afastando-a da creche, da escolinha, dos amigos, submetendo-a a procedimentos incômodos como exames, medicações, limitando inclusive sua atividade motora, representando um risco ao seu desenvolvimento.

Halpern e Figueiras ${ }^{14}$ chamam a atenção para uma nova morbidade, que se caracteriza pelos fatores de risco ao desenvolvimento psicossocial infantil.

Estes dados, sem dúvida, merecem atenção das equipes, especialmente para que se cumpram os objetivos de detecção precoce, prevenção e promoção de saúde, que fazem parte da proposta do Programa de Saúde da Família e da atenção primária em saúde.

Quanto às queixas apresentadas, a opção metodológica torna crítica à comparação dos resultados com os dados de literatura, uma vez que os trabalhos apontados tratam, predominantemente, de crianças entre zero e cinco anos e se baseiam no diagnóstico. ${ }^{1-}$ ${ }^{5}$ Entretanto, ao realizar tal comparação, nota-se que os dados divergem de outros estudos, pois diarréia e problemas respiratório-pulmonares não foram as principais causas de busca do serviço.

Por outro lado, as queixas de maior ocorrência na amostra estudada, foram problemas frequentemente associados com infecção das vias aéreas superiores e com infecções intestinais e verminoses.

É possível que alguns dos problemas apresentados pelas crianças sejam decorrentes de condições de moradia e higiene precárias, pois boa parte da população atendida nos serviços estudados reside em ruas de terra, casas sem laje, sem calefação e sem rede de esgoto, facilitando de certa forma todos os problemas que foram observados com maior frequência.

Como em outros estudos, as crianças mais novas mostraram-se mais suscetíveis a desenvolver problemas de saúde e, consequentemente, frequentaram mais os serviços.

Os resultados deste estudo destacam ainda, a ocorrência de problemas emocionais e comportamentais referidos pelas famílias, cuja frequência observada vai ao encontro dos dados da literatura que apon- 
tam que entre 10 e $20 \%$ das crianças apresentam algum problema de saúde mental. ${ }^{14}$ Entretanto, ressalta-se que em alguns momentos, a queixa apresentada poderia estar refletindo mais um desconhecimento da mãe sobre desenvolvimento do que um problema propriamente dito.

Vale salientar que se percebeu durante a coleta de dados uma diferença no procedimento de investigação e diagnóstico realizado pelo pediatra e pelo médico de família, sendo que os primeiros investigavam o comportamento e socialização da criança e os médicos de família investigavam prioritariamente a dimensão física. Este fato, inclusive pesou na decisão metodológica de se analisar inicialmente as queixas referidas.

Observou-se, por exemplo, que algumas vezes as queixas relatadas pela família eram ignoradas na avaliação e no diagnóstico, principalmente aquelas relacionadas a problemas emocionais e comportamentais ou de desenvolvimento.

Tal fato merece consideração, pois pode refletir a falta de preparo do médico na avaliação e detecção desses problemas.

Num estudo sobre o conhecimento dos profissionais das equipes de Saúde da Família sobre desenvolvimento infantil, as autoras concluíram que há uma deficiência por parte dos profissionais que atuam na atenção primária quanto ao conhecimento sobre desenvolvimento e comportamentos infantis, sendo que na prática a grande maioria não investiga, avalia ou orienta a família sobre estes aspectos. ${ }^{15}$

Os resultados deste trabalho apontam para a necessidade do treinamento continuado das equipes, especialmente no que tange ao desenvolvimento e saúde mental na infância e na sensibilização sobre a importância dos registros do serviço.

Ainda, é possível que atividades de educação para higiene, saúde e desenvolvimento infantis voltadas para as famílias pudessem ajudar a prevenir parte dos problemas encontrados e diminuir a frequência das crianças pequenas nos serviços, prevenindo também agravos ou desenvolvimento de psicopatologias ao longo da infância.

Pesquisas futuras poderiam verificar o impacto da educação continuada e dos grupos educativos na comunidade sobre a saúde da criança e funcionamento dos serviços, bem como a coerência entre o que motiva a busca dos serviços e a avaliação e diagnóstico efetivados. Também como já foi apontado, é preciso verificar porque alguns casos retornam com tanta frequência ao serviço e se estas crianças deveriam estar recebendo outro tipo de atenção à saúde.

\begin{abstract}
Paper design: prevalence study. Objective: To identify the major reasons that lead children aged 3 to 6 years to seek medical assistance at basic health care centers as well as to review their vaccination status and to determine at which frequency they attend to health care stations. Methods: A total of 370 medical records of children between 3 and 6 years old assisted at four Family Health Centers and one Basic Health Care Center were reviewed. Information regarding sex, age, number of consultations within the past 12 months, vaccination status and complaints reported at visits were collected. The complaints were organized in 28 categories according to the problem or affected organ. The frequency and percentage of all complaints were calculated as well as the indices of medical consultations per year and the vaccination coverage. Results: The most predominantly found complaints were cough, fever, nose problems, skin/scalp problems, gastric disorders, lack of appetite or loss of weight, emotional or behavioral disturbances, abdominal pains, respiratory and pulmonary disorders and ear problems. Only $3.2 \%$ of the children were not with their vaccination card updated and the number of consultations was 3.56 on average. Conclusions: Although the basic health care assistance tends to emphasize the aspects of prevention and heath promotion, there still is a privilege of the physical dimension at health promoters' sight and also in the search for assistance. The psychosocial aspects of health care should receive more attention.
\end{abstract}

Keywords: Childhood Health. Public Health. Morbidity. Health Profile. 


\section{Referências Bibliográficas}

1. Santos SR, Cunha AJLA, Gamba CM, Machado FG, Leal Filho JMM, Moreira NLM. Avaliação da assistência à saúde da muIher e da criança em localidade urbana da região Sudeste do Brasil. Rev Saúde Publica. 2000; 34:266-71.

2. Batista Filho M, Ferreira LOC. Um modelo para avaliação rápida da situação nutricional e de saúde de crianças e mães no "dia nacional de vacinação". Rev Bras Saúde Matern Infant. 2001; 1:145-54.

3. Caetano JRM, Bordin IAS, Puccini RF, Peres CA. Fatores associados à internação hospitalar de crianças menores de cinco anos, São Paulo, SP. Rev Saúde Publica. 2002; 36:28591.

4. Campos JJB, Forster AC, Soares DA. Estudo das condições de saúde das crianças do município de Londrina, PR, 1990. Semina Cienc Biol Saude. 1995; 16:224-33.

5. Puccini RF, Silva NN, Araújo NS, Pedroso GC, Silva EMK. Saúde infantil: condições de vida e utilização de serviços de saúde em área da região Metropolitana de São Paulo, 1996. Rev Bras Saúde Matern Infant. 2002; 2:143-55.

6. Monteiro CA. (org.). Velhos e novos males da saúde no Brasil: e evolução do país e de suas doenças. São Paulo: HucitecNupens/USP; 1995.

7. Osório MM. Fatores determinantes da anemia em crianças. J Pediatr 2002; 78:269-78.

8. Ministério da Saúde (Secretaria de Políticas de Saúde. Área da Saúde da Criança). Programas e projetos da saúde da criança: responsabilidades compartilhadas em benefício das crianças brasileiras. Rev Bras Saúde Matern Infant. 2002; 2:193-6.
9. Ministério da Saúde. Programa de Saúde da Família. 1994 [2004 Mar 25]. Disponível em: http://portal.saude.gov.br.

10. Goldbaum M, Gianini RJ, Novaes HMD , César CLG. Utilização de serviços de saúde em áreas cobertas pelo programa saúde da família (Qualis) no município de São Paulo. Rev Saúde Publica. 2005; 39:90-9.

11. Santana ML, Carmagnani MI. Programa de saúde da família no Brasil: um enfoque sobre seus pressupostos básicos, operacionalização e vantagens. Saúde Soc. 2001; 10:3354.

12. Pedrazzani ES, Cordeiro AMA, Furquim EC, Souza FF. Implantação de um banco de dados em vacinação: experiência desenvolvida em um projeto de integração. Rev Latinoam Enferm. 2002; 10:831-6.

13. Silva MAS, Gomes UA, Tonial SR, Silva RA. Cobertura vacinal e fatores de risco associados à não-vacinação em localidade urbana do nordeste brasileiro, 1994. Rev Saúde Publica. 1999; 33:147-56

14. Halpern R, Figueiras ACM. Influências ambientais na saúde mental da criança. J Pediatr. (Rio J.). 2004; 80(supl): S10410.

15. Figueiras ACM, Puccini RF, Silva EMK, Pedromônico MRM. Avaliação das práticas e conhecimentos de profissionais da atenção primária à saúde sobre vigilância do desenvolvimento infantil. Cad Saúde Pública. 2003;19:1691-9. 